\title{
A discrete version of Liouville's theorem on conformal maps
}

\author{
Ulrich Pinkall $^{1}$ (D) Boris Springborn ${ }^{1}$ (ID \\ Received: 8 September 2020 / Accepted: 26 March 2021 / Published online: 15 April 2021 \\ (c) The Author(s) 2021
}

\begin{abstract}
Liouville's theorem says that in dimension greater than two, all conformal maps are Möbius transformations. We prove an analogous statement about simplicial complexes, where two simplicial complexes are considered discretely conformally equivalent if they are combinatorially equivalent and the lengths of corresponding edges are related by scale factors associated with the vertices.
\end{abstract}

Keywords Discrete conformal map · Möbius transformation · Conformal flatness

Mathematics Subject Classification 53A30 - 52C26

\section{Introduction}

Liouville's theorem says that in dimension three and higher, conformal maps are Möbius transformations. More precisely:

Theorem (Liouville) If $U \subset \mathbb{R}^{n}$ is a domain and $n \geq 3$, then any sufficiently regular conformal map $f: U \rightarrow \mathbb{R}^{n}$ is the restriction of a Möbius transformation.

Liouville himself proved this theorem under the assumption that $f$ is four times continuously differentiable [16, Note VI, pp. 609-616]. Different modern proofs can be found, e.g., in the textbooks of Blaschke [1, §40, pp. 66f] and Dubrovin et al. [6, §15, pp. 138ff]. The regularity assumption can be weakened considerably. Gehring [7] and Reshetnyak [20] showed it is sufficient to assume that $f$ is in the Sobolev space $W_{n, l o c}^{1}(U)$, see also [3,12].

The purpose of this article is to extend the theorem in a different direction. We establish the following discrete version of Liouville's theorem for simplicial complexes (see Sect. 2 for precise definitions):

$凶$ Boris Springborn

boris.springborn@tu-berlin.de

https://page.math.tu-berlin.de/ springb

Ulrich Pinkall

pinkall@math.tu-berlin.de

https://page.math.tu-berlin.de/ pinkall

1 Institut für Mathematik, Technische Universität Berlin, Strasse des 17. Juni 135, 10623 Berlin, Germany 
Theorem 1 If $n \geq 3$, then two locally Delaunay discrete domains in $\mathbb{R}^{n}$ are discretely conformally equivalent if and only if they are Möbius equivalent.

Roughly, simplicial complexes are considered discretely conformally equivalent if they are combinatorially equivalent and the lengths of corresponding edges are related by scale factors associated with the vertices. They are considered Möbius equivalent if they are combinatorially equivalent and the vertex positions are related by a Möbius transformation.

One implication of the equivalence statement, "Möbius equivalence implies discrete conformal equivalence", holds for arbitrary simplicial complexes and for any dimension $n$ (see Sect. 3.1). The other implication, "discrete conformal equivalence implies Möbius equivalence", is only true for $n \geq 3$ and for a more restrictive class of simplicial complexes (see Sect. 3.3). In Definitions 2.3 and 2.4 we therefore define a locally Delaunay discrete domain to be a locally finite, full-dimensional simplicial complex that satisfies some additional conditions that are sufficient and necessary to deduce Möbius equivalence from discrete conformal equivalence in dimension three or greater.

The basic concepts are explained in Sect. 2. Section 3 is devoted to a proof of Theorem 1, which may in hindsight appear rather obvious. Theorem 1 and its proof suggest a necessary and sufficient condition for discrete conformal flatness. This, the connection between discrete conformal equivalence and hyperbolic geometry, and some open questions are discussed in Sect. 4.

A related approach to discretize the notion of conformality is via circle packings [25], or, in higher dimension, sphere packings. Cooper and Rivin's local rigidity theorem for sphere packings [5,8,21] and Xu's global version [26] state, roughly, that a 3-manifold triangulation and prescribed solid angles at the vertices determine a sphere packing uniquely (if it exists). An analogous statement may also hold in the setting of discrete conformal equivalence, roughly: A 3-manifold triangulation together with functions that assign a length to each edge and an solid angle to each vertex determine a discretely conformally equivalent triangulation with the given solid angles at the vertices uniquely (if it exists). It seems natural to expect that the analytic method of proof, based on a variational principle, also extends to this setting.

But our Theorem 1 is different in nature: It is not about the metric rigidity of piecewise flat closed manifolds, but about the Möbius rigidity of triangulated domains in $\mathbb{R}^{n}$. The method of proof is also very different: Rather than a variational principle, our proof of Theorem 1 relies on Cauchy's rigidity theorem for convex polyhedra and its higher dimensional generalization [18], which does all the hard work (see Sect. 3.3). The rest is essentially just setup. Cauchy's rigidity theorem will be applied to Möbius images of the links of interior vertices, with convexity ensured by the local Delaunay condition. This explains why the discrete Liouville theorem holds only in dimensions three and higher: In dimension two, the respective links are polygons, which are not rigid.

\section{Basic definitions}

In this article, a combinatorial isomorphism of simplicial complexes $K$ and $K^{\prime}$ in $\mathbb{R}^{n}$ is understood to be a bijection

$$
\phi: V \longrightarrow V^{\prime}
$$

between the vertex sets $V$ and $V^{\prime}$ of $K$ and $K^{\prime}$, respectively, such that for any subset $\left\{v_{0}, \ldots, v_{k}\right\} \subseteq V$ the simplex

$$
\left[v_{0}, \ldots, v_{k}\right] \subset \mathbb{R}^{n}
$$


is an element of $K$ if and only if the simplex

$$
\left[\phi\left(v_{1}\right), \ldots, \phi\left(v_{k}\right)\right] \subset \mathbb{R}^{n}
$$

is an element of $K^{\prime}$. Thus, a combinatorial isomorphism $\phi$ induces a bijection between the complexes $K$ and $K^{\prime}$, as well as a piecewise linear simplicial map between their carriers $|K|$ and $\left|K^{\prime}\right|$. Simplicial complexes are combinatorially equivalent if there exists a combinatorial isomorphism between their vertex sets.

Definition 2.1 (discrete conformal equivalence) Combinatorially equivalent simplicial complexes $K$ and $K^{\prime}$ in $\mathbb{R}^{n}$ are called discretely conformally equivalent with respect to a combinatorial isomorphism (1) if there exists a function

$$
u: V \longrightarrow \mathbb{R}
$$

such that the length of each edge $\left[v_{1}, v_{2}\right] \in K$ is related to the length of the corresponding edge $\left[\phi\left(v_{1}\right), \phi\left(v_{2}\right)\right] \in K^{\prime}$, by

$$
\left|\phi\left(v_{1}\right)-\phi\left(v_{2}\right)\right|=e^{\frac{1}{2}\left(u\left(v_{1}\right)+u\left(v_{2}\right)\right)}\left|v_{1}-v_{2}\right|,
$$

where $|\cdot|$ denotes the euclidean norm on $\mathbb{R}^{n}$. In other words, each edge length is scaled by the geometric mean of the scale factors $e^{u}$ attached to its vertices.

We say that $K$ and $K^{\prime}$ are discretely conformally equivalent if they are discretely conformally equivalent with respect to some combinatorial isomorphism.

This notion of discrete conformal equivalence appeared first in the four dimensional Lorentz-geometric context of the Regge calculus [22]. In the two-dimensional setting of surfaces, it has lead to a rich theory which is intimately connected with hyperbolic geometry [2,4,9-11,15,19,23] and useful in diverse applications, see, e.g., [13,14,24].

To fix ideas and introduce some notation, let us collect a few basic facts about Möbius transformations, beginning with the definition: A Möbius transformation of $\widehat{\mathbb{R}^{n}}$ is a composition of inversions in hyperspheres and reflections in hyperplanes, where

$$
\widehat{\mathbb{R}^{n}}=\mathbb{R}^{n} \cup\{\infty\}
$$

is the one-point compactification of $\mathbb{R}^{n}$.

A Möbius transformation preserves or reverses orientation, depending on whether it is a composition of an even or an odd number of inversions and reflections. The Möbius transformations of $\widehat{\mathbb{R}^{n}}$ form a Lie group $\operatorname{Möb}(n)$ of dimension $\frac{1}{2}(n+1)(n+2)$ which is isomorphic to the projectivized orthogonal group $\mathrm{PO}(n+1,1)$. Indeed, we may identify $\widehat{\mathbb{R}}^{n}$ with the $n$-dimensional unit sphere $S^{n} \subset \mathbb{R}^{n+1}$ via stereographic projection and consider $\mathbb{R}^{n+1}$ as the real projective space $\mathbb{R P}^{n+1}$ minus a projective hyperplane "at infinity". This identifies the Möbius group $\operatorname{Möb}(n)$ with the group $\mathrm{PO}(n+1,1)$ of projective transformations of $\mathbb{R P}^{n+1}$ that map the sphere $S^{n}$ to itself.

The group $\operatorname{Sim}(n)$ of similarity transformations of $\mathbb{R}^{n}$, i.e., of transformations of the form

$$
x \longmapsto \lambda A x+b,
$$

where

$$
\lambda \in \mathbb{R}_{>0}, \quad A \in O(n), \quad b \in \mathbb{R}^{n},
$$

is the subgroup of Möbius transformations that fix $\infty \in \widehat{\mathbb{R}^{n}}$ :

$$
\operatorname{Sim}(n)=\{T \in \operatorname{Möb}(n) \mid T(\infty)=\infty\} .
$$


Conversely, the Möbius group $\operatorname{Möb}(n)$ is generated by the similarity group $\operatorname{Sim}(n)$ together with one sphere inversion.

In Möbius geometry, a hypersphere in $\widehat{\mathbb{R}^{n}}$ is either a euclidean hypersphere in $\mathbb{R}^{n}$ or the union of a hyperplane in $\mathbb{R}^{n}$ with $\{\infty\}$. Möbius transformations map hyperspheres to hyperspheres. A Möbius transformation that is not a similarity transformation does not map simplices in $\mathbb{R}^{n}$ to simplices, except for zero-dimensional simplices, i.e., vertices. Two simplicial complexes are considered Möbius equivalent if their vertices are related by a Möbius transformation. More precisely:

Definition 2.2 (Möbius equivalence) Simplicial complexes $K$ and $K^{\prime}$ in $\mathbb{R}^{n}$ are called Möbius equivalent with respect to a combinatorial isomorphism $\phi: V \longrightarrow V^{\prime}$ if there is a Möbius transformation $T \in \operatorname{Möb}(n)$ such that

$$
\phi(v)=T(v) \text { for all vertices } v \in V .
$$

Simplicial complexes $K$ and $K^{\prime}$ are called Möbius equivalent if they are Möbius equivalent with respect to some combinatorial isomorphism.

The following Definitions 2.3 and 2.4 specify the extra assumptions under which we will show that discretely conformally equivalent simplicial complexes are Möbius equivalent. All of the conditions (i)-(iii) of Definition 2.3 and the local Delaunay condition of Definition 2.4 are necessary. It is easy (given the proof of Theorem 1 presented in Sect. 3) to construct examples showing that the implication may fail if any one of these conditions is not satisfied.

Definition 2.3 (discrete domain) A locally finite simplicial complex $K$ in $\mathbb{R}^{n}$ is called a discrete domain if it satisfies the following conditions:

(i) $K$ is $n$-dimensional and pure, i.e., $K$ contains only $n$-dimensional simplices and their faces.

(ii) Every $n$-dimensional simplex in $K$ has at least one interior vertex, i.e., a vertex contained in the interior of the carrier $|K|$.

(iii) The subgraph of the 1-skeleton of $K$ induced by the interior vertices is connected.

Definition 2.4 (local Delaunay condition) A discrete domain $K$ in $\mathbb{R}^{n}$ is called locally Delaunay if, for every $n$-simplex $\sigma \in K$, the open ball bounded by the circumsphere of $\sigma$ contains no vertices of $n$-simplices sharing a common $(n-1)$-face with $\sigma$.

Remark 2.5 Let $\sigma$ and $\sigma^{\prime}$ be two $n$-simplices in $\mathbb{R}^{n}$ that share a common $(n-1)$-face, say

$$
\sigma=\left[v_{0}, \ldots, v_{n}\right], \quad \sigma^{\prime}=\left[v_{1}, \ldots, v_{n+1}\right] .
$$

Then the following statements are equivalent:

- $v_{0}$ is contained in the open ball bounded by the circumsphere of $\sigma^{\prime}$.

- $v_{n+1}$ is contained in the open ball bounded by the circumsphere of $\sigma$.

Thus, the local Delaunay condition imposes one condition for every $(n-1)$-simplex in $K$ that is incident with two $n$-simplices.

To state the obvious, Theorem 1 refers to discrete conformal equivalence and Möbius equivalence with respect to the same combinatorial isomorphism, i.e., we will prove the following statement:

Theorem 1 (pedantic version) Suppose $n \geq 3, K$ and $K^{\prime}$ are locally Delaunay discrete domains in $\mathbb{R}^{n}$, and $\phi$ is a combinatorial isomorphism between $K$ and $K^{\prime}$. Then $K$ and $K^{\prime}$ are discretely conformally equivalent with respect to $\phi$ if and only if they are Möbius equivalent with respect to $\phi$. 


\section{Proof of Theorem 1}

\subsection{The easy implication: “Möbius equivalence implies discrete conformal equivalence"}

This implication holds for arbitrary simplicial complexes in $\mathbb{R}^{n}$ and for arbitrary dimension $n$. So let $K$ and $K^{\prime}$ be simplicial complexes in $\mathbb{R}^{n}$ and assume they are Möbius equivalent. We will show that they are discretely conformally equivalent.

If the Möbius transformation $T \in \mathrm{Möb}(n)$ relating $K$ and $K^{\prime}$ is a similarity transformation of $\mathbb{R}^{n}$, then $K$ and $K^{\prime}$ are obviously discretely conformally equivalent, because the relation (2) holds with a constant scale factor $e^{u}$.

If $T$ is the inversion in the unit sphere,

$$
T(x)=\frac{1}{|x|^{2}} x,
$$

then the identity

$$
|T(x)-T(y)|=\frac{1}{|x||y|}|x-y|,
$$

implies that $K$ and $K^{\prime}$ are discretely conformally equivalent. Indeed, in this case the relation (2) holds with $e^{u(v)}=|v|^{-2}$.

Since the similarity transformations and the inversion in the unit sphere generate the Möbius group, the implication holds for all $T \in \operatorname{Möb}(n)$.

\subsection{The equivalence of simplices}

In this section, we consider conformal equivalence and Möbius equivalence for pairs of simplices. In the next section, we will use the results to prove the harder implication, "discrete conformal equivalence implies Möbius equivalence."

Lemma 3.1 For $n$-simplices $\left[v_{0}, \ldots, v_{n}\right]$ and $\left[v_{0}^{\prime}, \ldots, v_{n}^{\prime}\right]$ in $\mathbb{R}^{n}$, the following statements are equivalent:

(i) There are real numbers $u_{0}, \ldots, u_{n}$ such that

$$
\left|v_{i}^{\prime}-v_{j}^{\prime}\right|=e^{\frac{1}{2}\left(u_{i}+u_{j}\right)}\left|v_{i}-v_{j}\right|
$$

for all two-element subsets $\{i, j\}$ of $\{0, \ldots, n\}$.

(ii) There is a Möbius transformation $T$ of $\widehat{\mathbb{R}^{n}}$ such that

$$
v_{i}^{\prime}=T\left(v_{i}\right) \text { for all } i \in\{0, \ldots, n\} .
$$

Section 3.1 proves the implication "(ii) $\Rightarrow$ (i)", so it remains to show the converse statement, "(i) $\Rightarrow$ (ii)". This is based on the following observations, which will also be useful by themselves:

Lemma 3.2 Assume condition (i) of Lemma 3.1 holds. Let $S_{v_{0}}$ and $S_{v_{0}^{\prime}}$ be the inversions in the spheres with radius 1 centered at $v_{0}$ and $v_{0}^{\prime}$, respectively, and let

$$
w_{i}=S_{v_{0}}\left(v_{i}\right), \quad w_{i}^{\prime}=S_{v_{0}^{\prime}}\left(v_{i}^{\prime}\right) .
$$

Then

$$
w_{0}=w_{0}^{\prime}=\infty
$$

and the $(n-1)$-simplices $\left[w_{1}, \ldots, w_{n}\right]$ and $\left[w_{1}^{\prime}, \ldots, w_{n}^{\prime}\right]$ are similar with scale factor $e^{-u_{0}}$. 
To show the implication "(i) $\Rightarrow$ (ii)" of Lemma 3.1 using Lemma 3.2, let $F$ be a similarity transformation of $\mathbb{R}^{n}$ mapping $w_{i}$ to $w_{i}^{\prime}$ for $i \in\{1, \ldots, n\}$, then

$$
T=S_{v_{0}^{\prime}} \circ F \circ S_{v_{0}}
$$

is a Möbius transformation satisfying (5). (Note that there are two such similarity transformations, one of which preserves orientation while the other reverses orientation.) This completes the proof of Lemma 3.1, assuming Lemma 3.2.

To prove Lemma 3.2, note that

$$
w_{i}=v_{0}+\frac{1}{\left|v_{i}-v_{0}\right|^{2}}\left(v_{i}-v_{0}\right) .
$$

for $i \in\{1, \ldots, n\}$, and a similar equation holds for $w_{i}^{\prime}$. Using the identity (3), one obtains

$$
\left|w_{i}-w_{j}\right|=\frac{1}{\left|v_{i}-v_{0}\right|\left|v_{j}-v_{0}\right|}\left|v_{i}-v_{j}\right|
$$

for $i, j \in\{1, \ldots, n\}$, and a similar equation for $\left|w_{i}^{\prime}-w_{j}^{\prime}\right|$. Now (4) implies

$$
\left|w_{i}^{\prime}-w_{j}^{\prime}\right|=e^{-u_{0}}\left|w_{i}-w_{j}\right|,
$$

and hence the simplices are similar with scale factor $e^{-u_{0}}$. This completes the proof of Lemma 3.2.

We will also use the following fact, the proof of which we leave to the reader:

Lemma 3.3 If there exists any Möbius transformation $T$ satisfying (5), then there exist exactly two of them, say, $T_{1}$ and $T_{2}$, of which one preserves orientation while the other reverses orientation, and which are related by

$$
T_{2}=T_{1} \circ C=C^{\prime} \circ T_{1},
$$

where $C$ and $C^{\prime}$ are the inversions in the circumspheres of the simplices $\left[v_{0}, \ldots, v_{n}\right]$ and $\left[v_{0}^{\prime}, \ldots, v_{n}^{\prime}\right]$, respectively.

\subsection{The harder implication: "discrete conformal equivalence implies Möbius equivalence"}

Let $K$ and $K^{\prime}$ be two locally Delaunay discrete domains in $\mathbb{R}^{n}$, where $n \geq 3$, and assume $K$ and $K^{\prime}$ are discretely conformally equivalent with respect to the combinatorial isomorphism $\phi$.

Note that $\phi$ may be orientation preserving or orientation reversing. We may assume without loss of generality that $\phi$ is orientation preserving. (If $\phi$ is orientation reversing, consider orientation preserving isomorphism between $K$ and a mirror image of $K^{\prime}$.)

Lemmas 3.1 and 3.3 say that for each $n$-simplex $\sigma \in K$, there is a unique orientation preserving Möbius transformation $T_{\sigma}$ such that $T_{\sigma}(v)=\phi(v)$ for every vertex $v \in \sigma$. Note that the assumptions about orientation ensure that $T_{\sigma}$ maps the inside of the circumsphere of $\sigma \in K$ to the inside of the circumsphere of $\phi(\sigma) \in K^{\prime}$.

It remains to show that these Möbius transformations $T_{\sigma}$ are in fact all equal. To this end, it is enough to show the equality for $n$-simplices in the star of an interior vertex, i.e., to show the following lemma:

Lemma 3.4 If $v$ is an interior vertex of $K$ and if $\sigma$ and $\tilde{\sigma}$ are two $n$-simplices contained in $\operatorname{star}(v)$, then $T_{\sigma}=T_{\tilde{\sigma}}$. 
Indeed, suppose Lemma 3.4 holds and $\sigma$ and $\tilde{\sigma}$ are any $n$-simplices of $K$. By assumption, both contain interior vertices of $K$, say $v$ and $\tilde{v}$. Furthermore, by assumption, there is a path from $v$ to $\tilde{v}$ in the 1 -skeleton of $K$ traversing only interior vertices. By induction on the length of the path, Lemma 3.4 implies that $T_{\sigma}=T_{\tilde{\sigma}}$.

The proof of Lemma 3.4 relies on Cauchy's rigidity theorem for convex polyhedra, applied to the link of $v$ after an inversion centered at $v$. Convexity follows from the following general observation:

Lemma 3.5 Let $K_{0}$ be a simplicial complex in $\mathbb{R}^{n}, n \geq 1$, with one interior vertex $v$ and $K_{0}=\operatorname{star}(v)$. Let $S$ be an inversion in some sphere centered at $v$, and apply it to the vertices of $\partial K_{0}$ to obtain a Möbius equivalent $(n-1)$-dimensional complex $P$. Then the following statements are equivalent:

(i) $K_{0}$ satisfies the local Delaunay condition.

(ii) $P$ is convex.

Proof (Lemma 3.5) Each $n$-simplex of $K_{0}$ corresponds to an $(n-1)$-dimensional face of $P$, and $S$ maps the closed ball bounded by the circumsphere of an $n$-simplex of $K_{0}$ to a closed halfspace in $\mathbb{R}^{n}$ bounded by the hyperplane of the respective face of $P$. Thus, the local Delaunay condition for $K_{0}$ is equivalent to a local convexity condition for $P$ involving adjacent faces, which is equivalent to the global convexity of $P$.

Now to show Lemma 3.4, let $v^{\prime}=\phi(v)$, and let $Q$ and $Q^{\prime}$ be the links of $v$ and $v^{\prime}$, respectively, i.e.,

$$
Q=\partial \operatorname{star}(v), \quad Q^{\prime}=\partial \operatorname{star}\left(v^{\prime}\right) .
$$

Let $S_{v}$ and $S_{v^{\prime}}$ be the inversions in the spheres with radius 1 centered at $v$ and $v^{\prime}$, respectively. Apply the inversions $S_{v}$ and $S_{v^{\prime}}$ to the vertices of $Q$ and $Q^{\prime}$, respectively, to obtain for each a Möbius equivalent polyhedron, $P$ and $P^{\prime}$. By Lemma 3.5, the local Delaunay condition for $K$ and $K^{\prime}$ implies that $P$ and $P^{\prime}$ are convex polyhedra in $\mathbb{R}^{n}$. As in Sect. 3.2, one sees that the facets of $P$ and $P^{\prime}$ are similar. By Cauchy's rigidity theorem for convex polyhedra and its higher dimensional generalization [18], there is a similarity transformation $F$ of $\mathbb{R}^{n}$ that maps $P$ to $P^{\prime}$. By the orientation assumption, $F$ is orientation preserving. Hence

$$
T=S_{v^{\prime}} \circ F \circ S_{v}
$$

is an orientation preserving Möbius transformation that maps $\operatorname{star}(v)$ to $\operatorname{star}\left(v^{\prime}\right)$. Therefore, $T=T_{\sigma}$ for all $\sigma \in \operatorname{star}(v)$. This proves Lemma 3.4 and hence the implication "discrete conformal equivalence implies Möbius equivalence", and this completes the proof of Theorem 1.

\section{Discrete conformal flatness and induced hyperbolic metric: concluding remarks, outlook and open questions}

The definition of discrete conformal equivalence (Definition 2.1) extends in an obvious way from simplicial complexes in $\mathbb{R}^{n}$ to triangulated piecewise euclidean manifolds, possibly with boundary, i.e., to manifolds that consist of euclidean simplices glued together along their facets. We propose the following notion of discrete conformal flatness:

Definition 4.1 A triangulated piecewise euclidean manifold is discretely conformally flat if the vertex star of every interior vertex is discretely conformally equivalent to a locally Delaunay discrete domain. 
Applying the same idea as in the proof of the discrete Liouville theorem, and in particular Eq. (6), one obtains the following result:

Theorem 2 A n-dimensional triangulated piecewise euclidean manifold is discretely conformally flat if and only if every interior vertex $v_{0}$ satisfies the following condition:

There exists a convex polyhedron in $\mathbb{R}^{n}$ that is combinatorially equivalent to the link of $v_{0}$ and whose edge lengths are

$$
\tilde{\ell}_{i j}=\frac{\ell_{i j}}{\ell_{0 i} \ell_{0 j}},
$$

Here, $\ell_{i j}$ denotes the length of the edge between two adjacent vertices $v_{i}, v_{j}$ of the triangulated piecewise euclidean manifold, and for vertices $v_{i}, v_{j}$ in the link of $v_{0}, \tilde{\ell}_{i j}$ denotes length of the corresponding edge of the convex polyhedron.

Note that for $n=2$, the condition on the vertex link is equivalent to the polyhedral inequalities for the $\tilde{\ell}_{i j}$, i.e., each $\tilde{\ell}_{i j}$ is larger than the sum of the others.

Note also that a connection between discrete conformal equivalence and hyperbolic geometry, which plays an important role in the theory in dimension two [2,23], extends to higher dimensions: If you interpret the circumsphere of a euclidean $n$-simplex as the boundary of $n$ dimensional hyperbolic space in the Beltrami-Klein model, this induces a hyperbolic metric on the simplex minus its vertices, turning the simplex into an ideal hyperbolic simplex. If you perform this construction on all simplices of a triangulated piecewise euclidean manifold, this induces a hyperbolic metric on the manifold with cusps at the vertices and cone-like singularities in the faces of codimension two. Just as in the two-dimensional setting, one can prove the following theorem:

Theorem 3 Two triangulated piecewise euclidean manifolds are discretely conformally equivalent if and only if they are isometric with respect to the induced hyperbolic metrics.

As in the two-dimensional setting [2,23], this observation suggests extending the definition of discrete conformal equivalence to triangulations that are not combinatorially equivalent:

Definition 4.2 (discrete conformal equivalence, extended) Two triangulated piecewise euclidean manifolds (which need not be combinatorially equivalent) are discretely conformally equivalent if they are isometric with respect to the induced hyperbolic metrics.

In the 2-dimensional setting, the known uniformization results $[11,23]$ show that any triangulated surface is discretely conformally flat, provided the notion of discrete conformal flatness is based on the extended notion of discrete conformal equivalence.

In dimensions 3 and higher, the situation more complicated. The induced hyperbolic metric will in general have cone-like singularities along faces of codimension 2 , even if the piecewise euclidean manifold is flat. The total dihedral angles at the faces of codimension 2 are discrete conformal invariants. Thus, any codimension-2-face whose hyperbolic cone angle is not equal to $2 \pi$ occurs in any discretely conformally equivalent triangulated piecewise euclidean manifold.

Maybe the most intriguing question opened by this line of research is how far the analogy between smooth and discrete conformal flatness extends. Consider for example the case of closed 3-dimensional manifolds $M$. The Chern-Simons functional $C S_{M}(g)$ is an $\mathbb{R} / \mathbb{Z}$-valued conformally invariant function on the space of Riemannian metrics $g$ on $M$, and the critical points of $C S_{M}$ are precisely the conformally flat metrics on $M$, see, e.g., [17]. Is there an analogous invariant on the set of discrete conformal classes whose critical points are exactly the discretely conformally flat classes? 
Acknowledgements This research was supported by the DFG SFB/TR 109 "Discretization in Geometry and Dynamics".

Funding Open Access funding enabled and organized by Projekt DEAL.

Open Access This article is licensed under a Creative Commons Attribution 4.0 International License, which permits use, sharing, adaptation, distribution and reproduction in any medium or format, as long as you give appropriate credit to the original author(s) and the source, provide a link to the Creative Commons licence, and indicate if changes were made. The images or other third party material in this article are included in the article's Creative Commons licence, unless indicated otherwise in a credit line to the material. If material is not included in the article's Creative Commons licence and your intended use is not permitted by statutory regulation or exceeds the permitted use, you will need to obtain permission directly from the copyright holder. To view a copy of this licence, visit http://creativecommons.org/licenses/by/4.0/.

\section{References}

1. Blaschke, W.: Vorlesungen über Differentialgeometrie und geometrische Grundlagen von Einsteins Relativitätstheorie. I: Elementare Differentialgeometrie. Springer, Berlin (1921)

2. Bobenko, A.I., Pinkall, U., Springborn, B.: Discrete conformal maps and ideal hyperbolic polyhedra. Geom. Topol. 19(4), 2155-2215 (2015). https://doi.org/10.2140/gt.2015.19.2155

3. Bojarski, B., Iwaniec, T.: Another approach to Liouville theorem. Math. Nachr. 107, 253-262 (1982)

4. Bücking, U.: $C^{\infty}$-convergence of conformal mappings for conformally equivalent triangular lattices. Results Math. 73(2), 21 (2018). https://doi.org/10.1007/s00025-018-0845-2

5. Cooper, D., Rivin, I.: Combinatorial scalar curvature and rigidity of ball packings. Math. Res. Lett. 3(1), 51-60 (1996)

6. Dubrovin, B.A., Fomenko, A.T., Novikov, S.P.: Modern Geometry-Methods and Applications. Part I, 2nd edn. Springer, Berlin (1992)

7. Gehring, F.W.: Rings and quasiconformal mappings in space. Trans. Am. Math. Soc. 103, 353-393 (1962)

8. Glickenstein, D.: A maximum principle for combinatorial Yamabe flow. Topology 44(4), 809-825 (2005)

9. Gu, D., Luo, F., Wu, T.: Convergence of discrete conformal geometry and computation of uniformization maps. Asian J. Math. 23(1), 21-34 (2019)

10. Gu, X., Guo, R., Luo, F., Sun, J., Wu, T.: A discrete uniformization theorem for polyhedral surfaces II. J. Differ. Geom. 109(3), 431-466 (2018)

11. Gu, X.D., Luo, F., Sun, J., Wu, T.: A discrete uniformization theorem for polyhedral surfaces. J. Differ. Geom. 109(2), 223-256 (2018)

12. Iwaniec, T., Martin, G.: Quasiregular mappings in even dimensions. Acta Math. 170(1), 29-81 (1993)

13. Koehl, P., Hass, J.: Automatic alignment of genus-zero surfaces. IEEE Trans. Pattern Anal. Mach. Intell. 36(3), 466-478 (2014)

14. Konaković, M., Crane, K., Deng, B., Bouaziz, S., Piker, D., Pauly, M.: Beyond developable: computational design and fabrication with auxetic materials. ACM Trans. Graph. 35(4), 89:1-89:11 (2016)

15. Luo, F.: Combinatorial Yamabe flow on surfaces. Commun. Contemp. Math. 6(5), 765-780 (2004)

16. Monge, G., Liouville, J.: Application de l'analyse à la géométrie. Bachelier, Paris (1850)

17. Moroianu, S.: The cotton tensor and Chern-Simons invariants in dimension 3: an introduction. Bul. Acad. Ştiinţe Repub. Mold. Mat. 2(78), 3-20 (2015). (arXiv:1509.05156 [math.DG])

18. Pak, I.: Lectures on Discrete and Polyhedral Geometry. Manuscript (2010). http://www.math.ucla.edu/ $\sim$ pak/book.htm. Retreived 06 Sept 2019

19. Prosanov, R.: Ideal polyhedral surfaces in Fuchsian manifolds. Geom. Dedicata 206, 151-179 (2020)

20. Rešetnjak, J.G.: Liouville's conformal mapping theorem under minimal regularity hypotheses. Sibirsk. Mat. Ž. 8, 835-840 (1967)

21. Rivin, I.: An extended correction to "combinatorial scalar curvature and rigidity of ball packings," (by D. Cooper and I. Rivin). arXiv:math/0302069v2 (2003)

22. Roček, M., Williams, R.M.: The quantization of Regge calculus. Z. Phys. C 21(4), 371-381 (1984)

23. Springborn, B.: Ideal hyperbolic polyhedra and discrete uniformization. Discrete Comput. Geom. 64(1), 63-108 (2020)

24. Springborn, B., Schröder, P., Pinkall, U.: Conformal equivalence of triangle meshes. ACM Trans. Graph. 27(3), 11 (2008)

25. Stephenson, K.: Introduction to Circle Packing. Cambridge University Press, Cambridge (2005) 
26. Xu, X.: On the global rigidity of sphere packings on 3-dimensional manifolds. J. Differ. Geom. 115(1), 175-193 (2020)

Publisher's Note Springer Nature remains neutral with regard to jurisdictional claims in published maps and institutional affiliations. 